\title{
ELASTICITY OF HUMAN VOCAL FOLDS MEASURED IN VIVO USING COLOR DOPPLER IMAGING
}

\author{
Tzu-Yu Hsiao, ${ }^{*}$ Chung-Li Wang, ${ }^{\dagger}$ Chiung-Nien Chen, ${ }^{\dagger}$ Fon-Jou HsieH $^{\dagger}$ and \\ YIO-Wha SHAU \\ *Departments of Otolaryngology, and Diagnostic Ultrasound, National Taiwan University Hospital, Taipei, Taiwan; \\ and Institute of Applied Mechanics, National Taiwan University, Taipei, Taiwan
}

(Received 25 March 2002; in final form 23 May 2002)

\begin{abstract}
Noninvasive measurement of human vocal fold (VF) vibratory length was made during normal phonation to calculate the corresponding elasticity. A fixed-ends rubber string phantom that was driven by a vibrating motor was built to simulate the horizontal VF movement. The vibratory artefact of color Doppler imaging (CDI) was used to characterize and quantify the high-frequency tissue vibration with small amplitude. Because the frequency and the length of vibrating VFs were obtained simultaneously, the stress-strain relation and the Young's moduli of the VFs could be calculated. For the six vocally normal adult volunteers $(3 \mathrm{M}, 3 \mathrm{~F}$, ages from 19 to 51 years old), the effective vibrating lengths of the vocal fold in low pitch were about 1.4 to 1.6 $\mathrm{cm}$ and 1.3 to $1.5 \mathrm{~cm}$ for men and women, respectively. The VFs lengths extended to about 1.7 to $1.8 \mathrm{~cm}$ in pitch over an octave higher and the stress-strain relation was nonlinear. However, in the range of lower pitch, the VF stress was relatively linear with respect to the strains and the Young's moduli were about 30 to $120 \mathrm{kPa}$ in men and 120 to $300 \mathrm{kPa}$ in women. (E-mail: ywshau@spring.iam.ntu.edu.tw) @ 2002 World Federation for Ultrasound in Medicine \& Biology.
\end{abstract}

Key Words: Vocal fold length, Fundamental frequency, Elasticity, Color Doppler image, Voice, Phonation.

\section{INTRODUCTION}

The vocal folds (VFs) are known to vibrate at their natural frequencies as the airflow passes through the glottis, and the vibration frequency of the VF determines the pitch of the voice produced (van den Berg 1958). The configuration of the glottis under the action of laryngeal muscles and the subglottal pressure dominates the natural frequency of the VFs (Hirano 1975; Titze et al. 1987; Titze 1989; Hsiao et al. 1994). So far, little is known with respect to the VF biomechanical properties in vivo in controlling its vibratory movements (Titze 1994). VF vibration during phonation is a very complex motion. The mucosal wave, which is the summation of sequential horizontal tissue displacement from the infraglottic to the supraglottic extent, propagates vertically from the lower to the upper margin of the VFs (Titze 1988; Titze et al. 1993). In the horizontal view, the VFs are fixed to the anterior commissure and the vocal process of arytenoid cartilage on both ends. Microscopically, the VF being a

Address correspondence to: Yio-Wha Shau, Ph.D., Biophysics and Gasdynamics Lab., Institute of Applied Mechanics, National Taiwan University Taipei 106. E-mail: ywshau@spring.iam.ntu.edu.tw multilayered structure, can be divided into the cover and the body (Hirano 1974). The cover (including the mucosa and the superficial layer of lamina propria) is much more pliable than the body (consisting of the vocal muscle and ligament), so the vibration of VFs is confined mostly in the cover (Saito et al. 1983). The elastic properties of the VFs, especially those of the cover, play an important role in the mechanisms of pitch control. Because the horizontal structure of the VFs resembles a bilaterally fixed string (Titze 1994), the natural frequency $(f)$ of string vibration is known to be:

$$
f=\frac{1}{2 L} \sqrt{\frac{T}{\rho}},
$$

where $T$ is the longitudinal tension, $\rho$ is the mass length density, and $L$ is the length of the string. Therefore, the change of intrinsic tension with respect to the elongation of the VF (i.e., VF stiffness) can be related to the change of frequency during phonation.

In the previous studies, the VF mechanical properties were mostly investigated in vitro (van den Berg and Tan 1959; Perlman et al. 1984; Alipour-Haghighi and 
Titze 1991; Kakita et al. 1983; Perlman and Titze 1988; Haji et al. 1992a, 1992b; Min et al. 1995). In vivo measurements of VF elasticity (human or canine) were conducted under general anesthesia and the VFs were in resting status (Tran et al. 1993; Yumoto and Kadota 1997; Tanaka and Hirano 1990; Berke 1992; Berke and Smith 1992).

The quantification of VF mechanical properties in motion is a critical step to understand the phonation physiology. Medical ultrasound (US) provides an ideal noninvasive method for assessing the biomechanical properties of the VFs because it is easy to perform and minimally disturbs the voice production. In our previous study, the color Doppler imaging (CDI) of the VFs vibration has been characterized and quantified (Shau et al. 2001). The vibrating amplitude, frequency, mass density and the acoustic impedance of the VFs are found to dominate the contour of the CDI artefacts. The vibratory portion of the VF can be identified by the color contour. In this present study, we applied the US to measure the longitudinal length of the vocal folds $(L)$ in motion. The voicing frequency $(f)$ during phonation was also detected simultaneously. With the help of standing-wave phantom, the CDI was shown feasible for quantifying the elastic properties of human VFs in vivo.

\section{MATERIALS AND METHODS}

\section{Ultrasound identification of the vocal fold}

A commercially available, high resolution US scanner (HDI-5000, ATL, Bothell, WA) with a 5- to $12-\mathrm{MHz}$ linear-array transducer (L12 to $538 \mathrm{~mm}$, ATL) was used in this study. The imaging area was $3.8 \mathrm{~cm}$ wide and 4.8 $\mathrm{cm}$ in depth. The frame rate in B-mode was about $25 \mathrm{~Hz}$. In the color mode, the pulse-repetition rate was 10,000 $\mathrm{Hz}$ and the measuring velocity range was set at 0 to 128.3 $\mathrm{cm} / \mathrm{s}$ with baseline offset, which resulted in a frame rate of about $7 \mathrm{~Hz}$. The viewing window in the color mode is high enough to detect the vibratory part of the VF in most of the cases (Hsiao et al. 2001). The US scan head was placed horizontally at the midline of the thyroid cartilage lamina on one side (Fig. 1a). By changing the scanner direction rostrally and caudally, the interface between true vocal fold mucosa and the air column in the larynx can be identified as a white line. The white line is at the lateral position of the larynx in quiet respiration and the attachment to the anterior commissure can be clearly seen (Fig. 1b). During phonation, both VFs adduct and contact at the midline of the glottis. The white line becomes obscure (Miles 1989; Hsiao et al. 2001). In voicing, a regular moving pattern of the US artefact between the mucosa and the air column can also be identified (Fig. 1c). In color mode, color strip artefacts generated from the vibration of the interface between the mucosa and the air column can be appreciated in a specific contour (Fig. 1d).

Computer simulation of the color strip formation of VF vibration in $C D I$

To understand the formation of vibratory color strips in CDI, the numerical simulation for a vibrating string under US line-scanning was performed using MATLAB (Mathworks, Inc., Natick, MA). Generally, the displacement of a vibrating string, $y(x, t)$ that behaves as a standing sinusoidal wave can be approximated by

$$
y(x, t)=A o \sin (2 \pi x / \lambda) \cos (2 \pi f t)
$$

where $A o$ is the maximum of the vertical displacement, $f$ is the vibrating frequency, and $\lambda$ is the wavelength. The displacement velocity would be the instantaneous time rate of change of the displacement, $\partial y(x, t) / \partial t$ :

$v(x, t)=\partial y(x, t) / \partial t=-2 \pi f A o \sin (2 \pi x / \lambda) \sin (2 \pi f t)$

However, because the US beam scans line-by-line across the viewing window as the string vibrates at a higher frequency continuously (Fig. 2a), the displacement velocity observed by the US scanning line will encompass numbers of zeros as the line marches in the $x$ direction. The number of red-blue strip pairs approximately reflects the ratio of the vibrating frequency of the string to the CDI frame rate (Fig. 2b).

Color Doppler imaging for bilaterally fixed-ends vibrating string

To investigate the fixed-ends vibrating tissue in CDI (e.g., the VF in the horizontal plane), a vibrating rubber string phantom of length $1.7 \mathrm{~cm}$ was built. With both ends fixed, the rubber string was pierced at the midpoint by a needle, and a vibrating motor was connected to the needle to generate the vibratory movement. The phantom was immersed in water at about 6 to $7 \mathrm{~cm}$ above the bottom surface to reduce the interference of US reflection, and the US transducer was placed at about $2 \mathrm{~cm}$ above the string. The motor generated 60 to $100 \mathrm{~Hz}$ up and down vibrations, depending on the voltage supplied. To simulate the actual relation between the US scanner and the vibrating VF, the US scanner and the rubber string were placed to incline at an angle of about $30^{\circ}$ (Fig. 3a).

In CDI, the vibrating rubber string formed a grey shadow, and the vibratory movements of the string caused a regular pattern of color strips underneath the string (Fig. 3b). The depth of the color strips underneath the string was found to correlate with the vibrating am- 

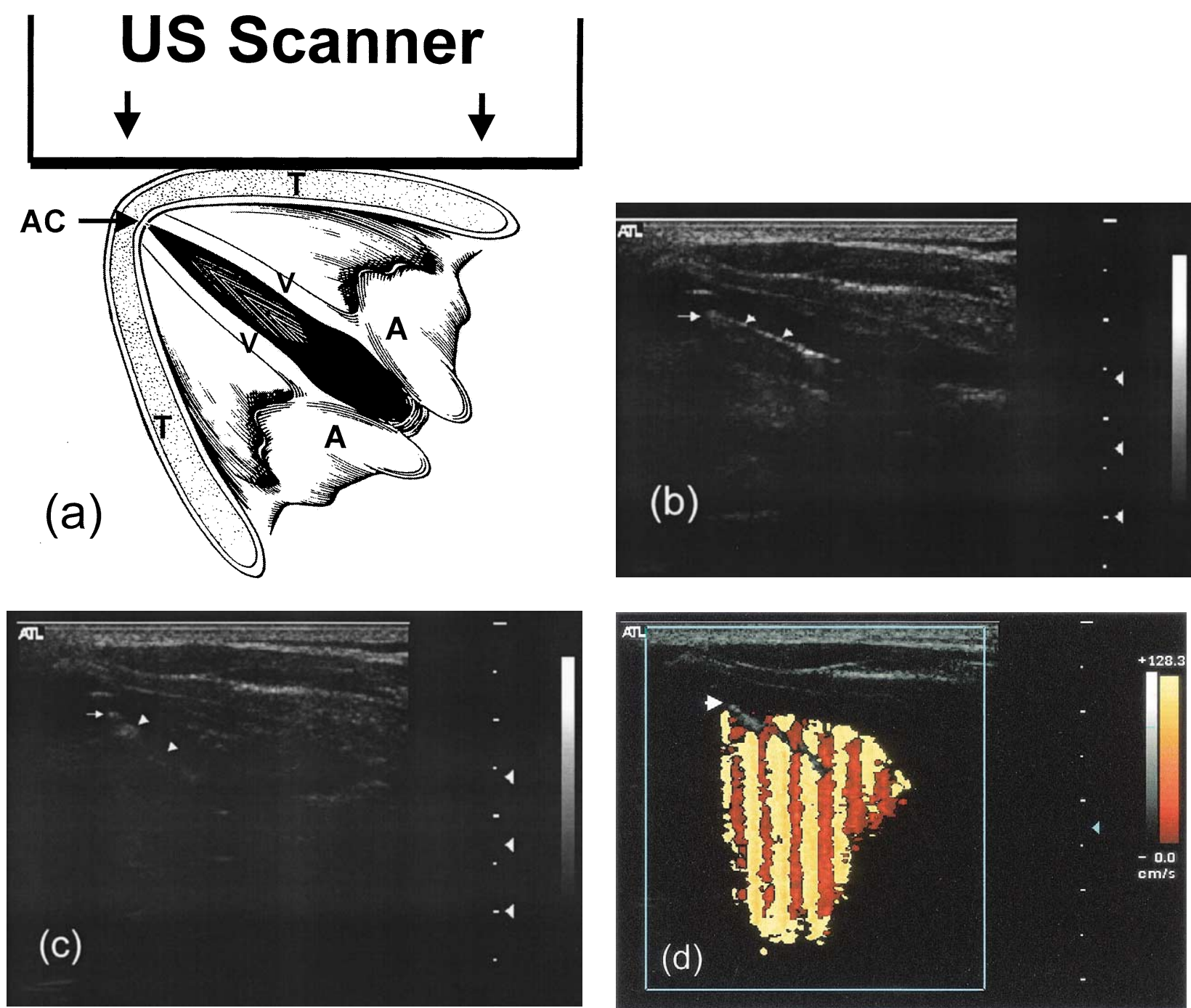

Fig. 1. (a) Schematic demonstration of the relation between the US scanner and the VF in the horizontal plane. $\mathrm{T}=$ thyroid cartilage; $\mathrm{AC}=$ anterior commissure; $\mathrm{A}=$ arytenoid cartilage; $\mathrm{V}=$ vocal fold. (b) In B-mode, the white line artefact of the interface between true VF mucosa and the air column in the larynx can be identified (arrowheads). (c) During VF vibration, the white line becomes obscure (arrowheads). (d) In CDI, regular color strips appear in a specific contour. Arrow in (b) to (d) $=$ The attachment of VF to the anterior commissure.

plitude of the string. The midpoint of the string, where the maximum vibrating amplitude occurred, generated the longest color strip in the CDI. The depth of the strips tapered to both fixed ends. Although there were some motion artefacts beyond the limit of the vibrating string, the boundaries of the string could be clearly delineated by the contour of the color map (Fig. 3b). The right boundary of the string was obtained from the crossing point of the grey string line and the color contour (Fig. $3 b)$. Using the $\mathrm{C}++$ image-processing software that was developed earlier (Shau et al. 1999), we measured the length of the string from its midpoint (metallic needle) to the right extreme of the string vibrating at $61 \mathrm{~Hz}$.
Color Doppler imaging in voicing human subjects

A total of 6 healthy adults ( 3 men, 3 women), ages ranging from 19 to 51 years old, with informed consent, were involved in the study. The subjects without voice disorders were asked to produce a sustaining long vowel in modal voice. The voice intensity was soft and kept in about 60 to $70 \mathrm{~dB}$. At least 8 to 12 different pitches were recorded by following the electronic keyboard from the lowest key obtainable up to over an octave. During phonation, about 15 CDI frames of the VFs were taken in each pitch. The voice signals were detected with a precision microphone (ECM-672, SONY, Tokyo, Japan) and the fundamental frequency of the voice $(f)$ was 

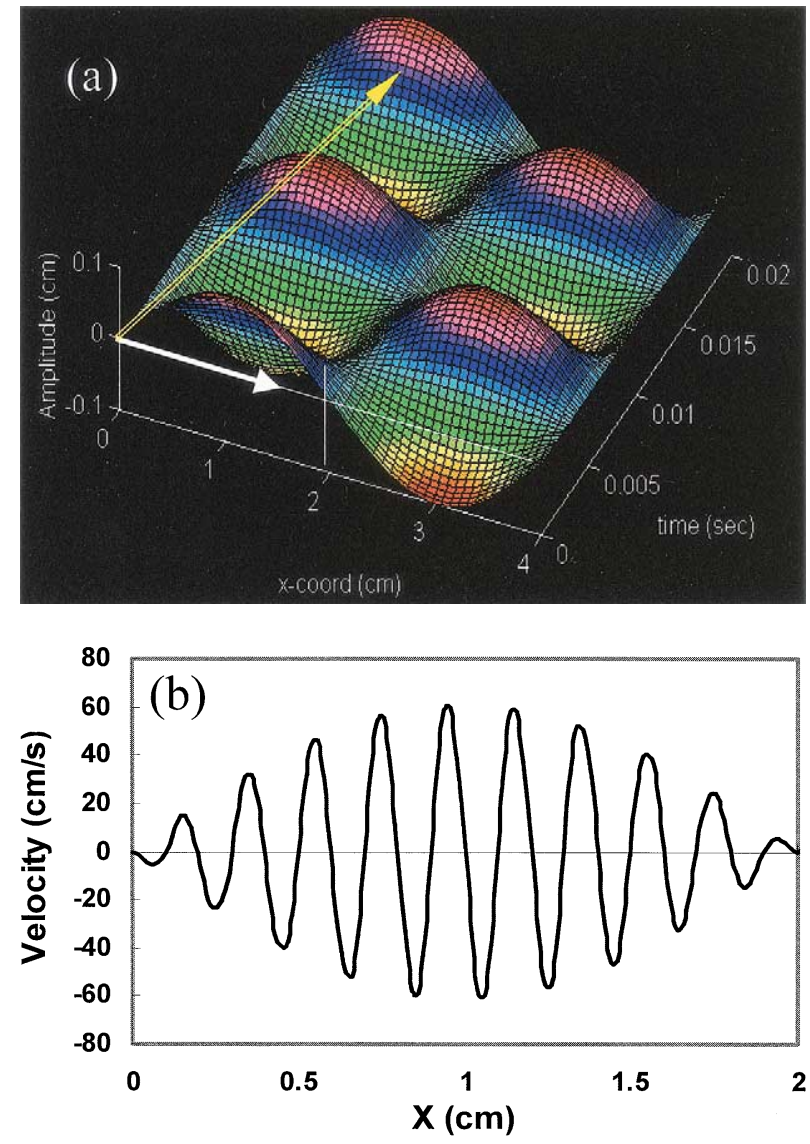

Fig. 2. (a) Vibratory string displacement, $y(x, t)$, for a standing wave given by the MATLAB simulation. The US line scan direction moved along the $\mathrm{x}$-direction (white arrow) as it marched in time (yellow arrow). (b) Local vibratory velocity observed instantaneously by the US scan line as it swept through the viewing window. $f=100 \mathrm{~Hz} ; A o=0.1 \mathrm{~cm}, \lambda=$ $4 \mathrm{~cm}$ and CDI frame rate of $10 \mathrm{~Hz}$ with viewing window size of $2 \mathrm{~cm}$.

measured and displayed simultaneously on the digital oscilloscope (TDS-224, Tektronix, Beaverton, OR) that was equipped with fast Fourier transform (FFT) module (TDS2 MM). All the examinations were performed on the right $\mathrm{VF}$, with one exception of one man (47-yearold), who was recorded on both VFs.

The anterior end of the vocal fold, the attachment to the anterior commissure, can be clearly visualized in the CDI pictures. The posterior end of the vibrating vocal fold can be obtained using the same method as in the phantom experiment (Fig. 4). The distance between these two points was the length of the VF during vibration. For each voicing frequency $(f)$, about 15 frames were taken for averaging of vocal cord length to minimize the error of measurement.
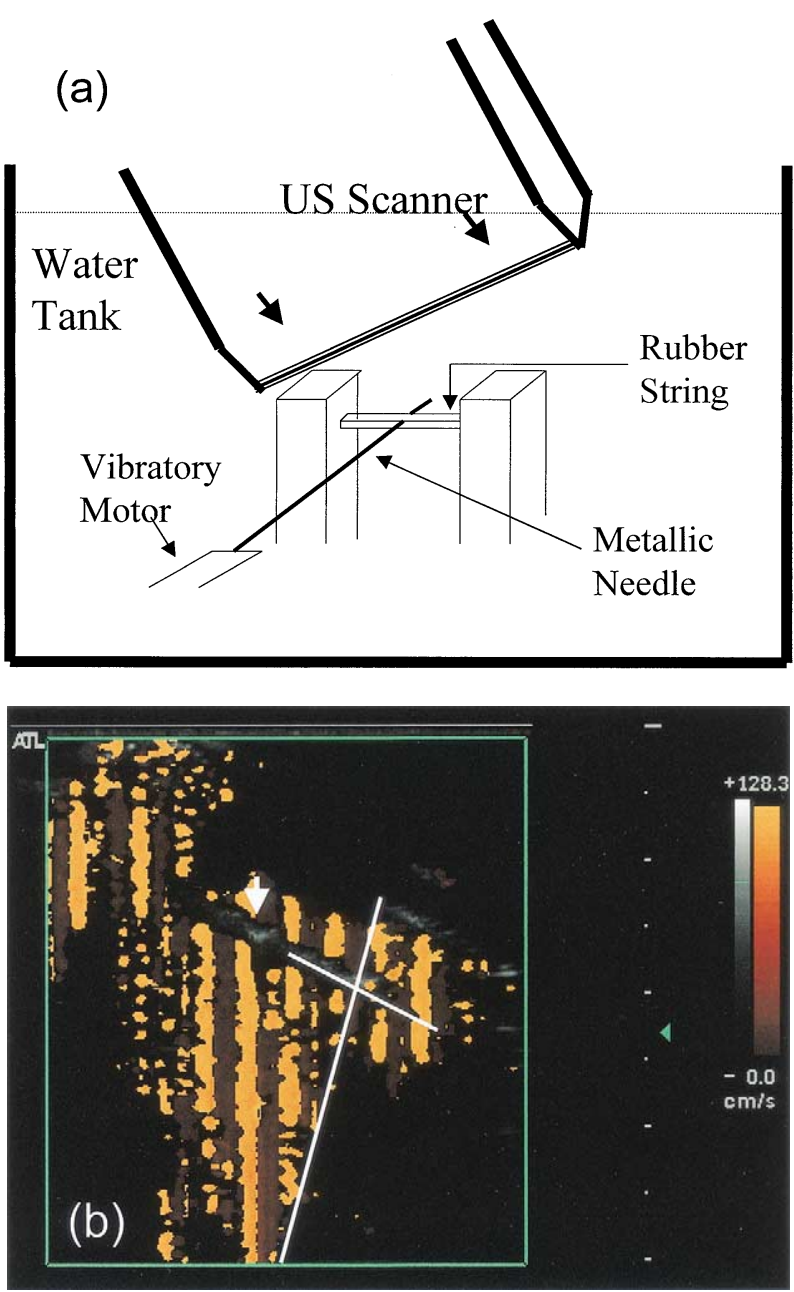

Fig. 3. (a) Schematic of the vibrating rubber string phantom with both ends fixed. (b) CDI of the vibrating string. The right end of the string can be determined by the line crossings. Arrow $=$ The midpoint of the string.

Calculation of the tension and stress of vocal fold during vibration

The tension of the VF during vibration can be obtained by using eqn (1) based on the voicing frequency $(f)$ and the length of the $\operatorname{VF}(L)$ measured simultaneously. The mass length density $(\rho=\mathrm{m} / \mathrm{L})$ can also be calculated based on the given VF mass $(m)$ and the length $(L)$. According to the previous report (Perlman and Durham 1987), the mass $(m)$ of the human VF cover is about $0.169 \mathrm{gm}$ in men, $0.124 \mathrm{gm}$ in women, and average density of the tissue $(\mu)$ is $1.02 \mathrm{gm} / \mathrm{cm}^{3}$. The mass of the VF cover along the vibration margin was found to be relatively constant in microscopic study (Hirano 1975). The stress of the vocal fold $(\sigma)$ during vibration can be derived from the tension $(T)$ and the cross-section area $(A$ $=\mathrm{m} / \mu \mathrm{L})$, where 

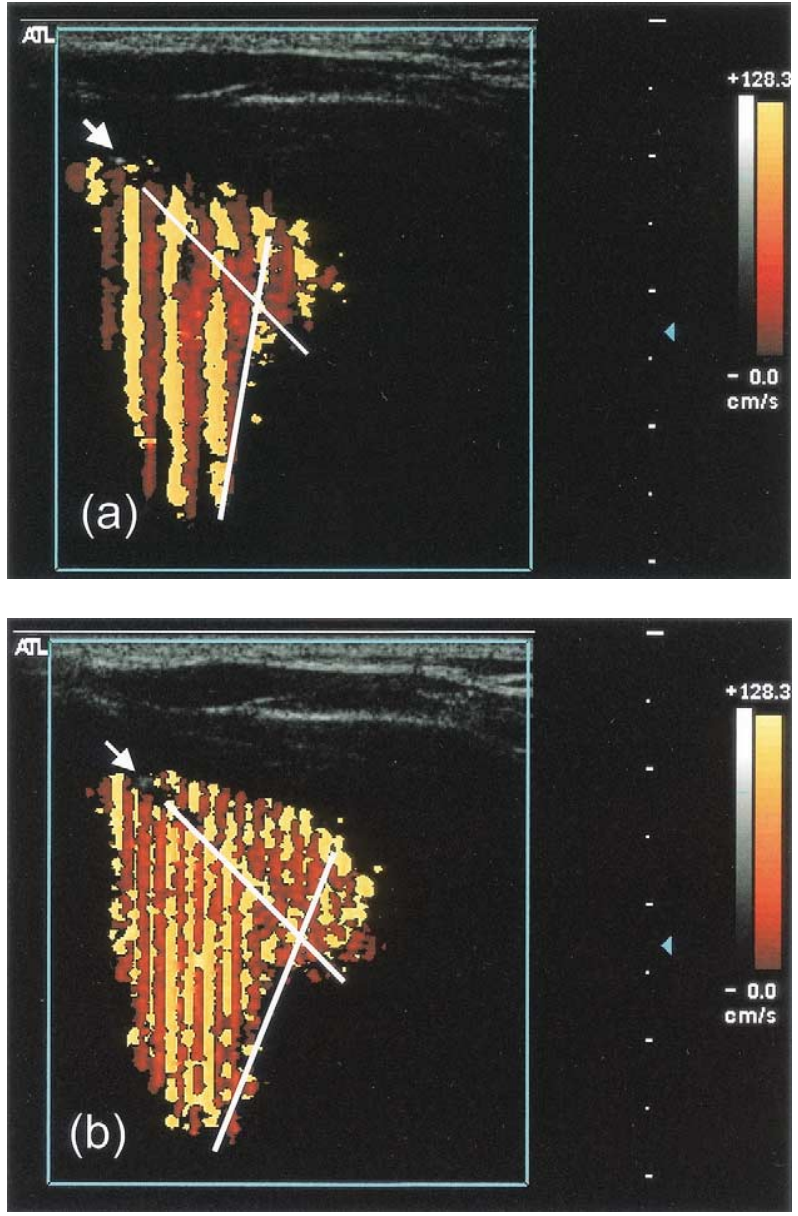

Fig. 4. CDI of the VFs vibration in one of the men (M1R) at the fundamental frequency of (a) $80 \mathrm{~Hz}$ and (b) $172 \mathrm{~Hz}$. The length of the vibrating VF can be measured from the anterior commissure (arrow) to the posterior end of the VF (the lines crossing point); $1.44 \mathrm{~cm}$ in (a) and $1.72 \mathrm{~cm}$ in (b).

$$
\sigma=T / A=T \mu \mathrm{L} / \mathrm{m}
$$

The strain $(\varepsilon)$ of the VF during phonation is given by normalizing the VF elongation $\left(\Delta L=L-L_{o}\right)$ by its length at the lowest frequency of phonation $\left(L_{o}\right)$.

$$
\epsilon=\left(L-L_{\mathrm{o}}\right) / / L_{\mathrm{o}}=\Delta L / L_{\mathrm{o}}
$$

The Young's modulus $(E)$ of the VF is simply given by:

$$
E=d \sigma / d \epsilon
$$

\section{RESULTS}

To verify the accuracy of US length measurement, we averaged the lengths of vibrating rubber string from $15 \mathrm{CDI}$ frames (from the midpoint to the right end) and

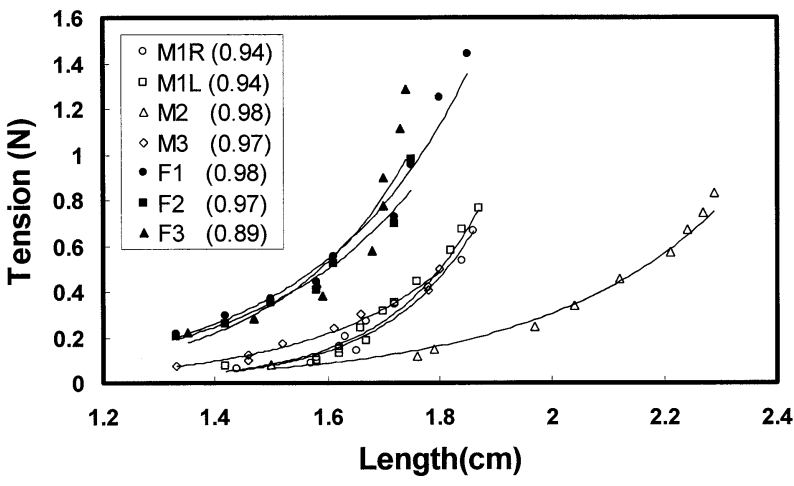

Fig. 5. Length-tension relation of six subjects (seven fitted curves). The numbers in the parenthesis are the regression coefficient. M1L = man \#1, left vocal fold.

the result was $0.88 \pm 0.02 \mathrm{~cm}$, which deviated from the actual length $(0.85 \mathrm{~cm})$ by about $3 \%$. Using the same technique, the vibrating length of the VFs was measured for every pitch of phonation. The effective vibrating length of the VF was about 1.4 to $1.6 \mathrm{~cm}$ in low-pitch voices in men and was about 1.3 to $1.5 \mathrm{~cm}$ in women. The length extended to about 1.7 to $1.8 \mathrm{~cm}$ in both men and women in pitch over an octave higher. Figure 5 shows the tension of the VF vs. the active length of the VF. The tension-length relation was nonlinear and the curves were well fitted to exponential functions. Almost all the regression coefficients were greater than 0.9 . The tension of the VF for women was greater than that for the men. The value of tension ranged from about 0.06 to 0.8 $\mathrm{N}$ in the frequency range of 80 to $246 \mathrm{~Hz}$ in men, and it was about 0.22 to $1.4 \mathrm{~N}$ in the frequency range of 176 to $396 \mathrm{~Hz}$ in women.

The data derived from both VFs of a man revealed almost identical curves. Using the vocal fold length of the lowest pitch $\left(L_{o}\right)$ as a reference, the stress-strain relation is plotted in Fig. 6 and, again, the data were well

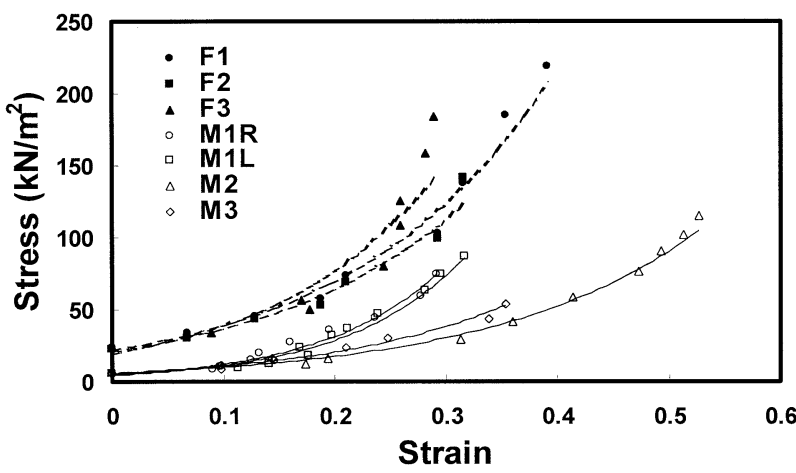

Fig. 6. Stress-strain curves derived from the data in Fig. 3 with the VF length of the lowest vibration frequency as a reference. 
Table 1. The constant values and regression coefficient of fitted stress-strain exponential curves in Fig. 6

\begin{tabular}{lccc}
\hline & $a$ & $b$ & $\mathrm{R}^{2}$ \\
\hline M1L & 4.68 & 9.49 & 0.95 \\
M1R & 4.36 & 9.41 & 0.95 \\
M2 & 5.88 & 5.47 & 0.98 \\
M3 & 5.87 & 6.24 & 0.98 \\
F1 & 22.13 & 5.72 & 0.99 \\
F2 & 21.15 & 5.55 & 0.98 \\
F3 & 19.71 & 6.79 & 0.92 \\
\hline
\end{tabular}

$\sigma=a e^{b \epsilon}$.

fitted to exponential curves $\left(\sigma=a e^{b \varepsilon}\right)$. The curve-fitted values of exponential power $b$ ranged from 5.47 to 9.49 in men and 5.56 to 6.79 in women, as in Table 1. The Young's modulus of the VF defined by the ratio of stress to the strain can be calculated from the slope of stressstrain curves and is plotted in Fig. 7. Again, the stressstrain relation was nonlinear, and the Young's modulus of the VF varied with VF length. However, in low-strain portions of these curves $(<10 \%)$, they were relatively linear. The values of the Young's moduli in the linear region were about 30 to $120 \mathrm{kPa}$ in men and 120 to 300 $\mathrm{kPa}$ in women.

\section{DISCUSSION}

The VF vibration is purely a mechanical issue (Titze 1994). Based on the concept of cover-body theory, the oscillation part is largely confined to the cover of the VFs (Hirano 1974; Saito et al. 1983). The coordinated action of all the intrinsic and extrinsic laryngeal muscles, especially the cricothyroid (CT) and thyroarytenoid (TA) muscles primarily determines the mechanical characteristics of the VF cover (Hirano 1975; Titze et al. 1987). The action of CT elongates the VFs, increases the tension and thins the edge. The TA, on the contrary, shortens, slackens and thickens the VFs. The muscular action

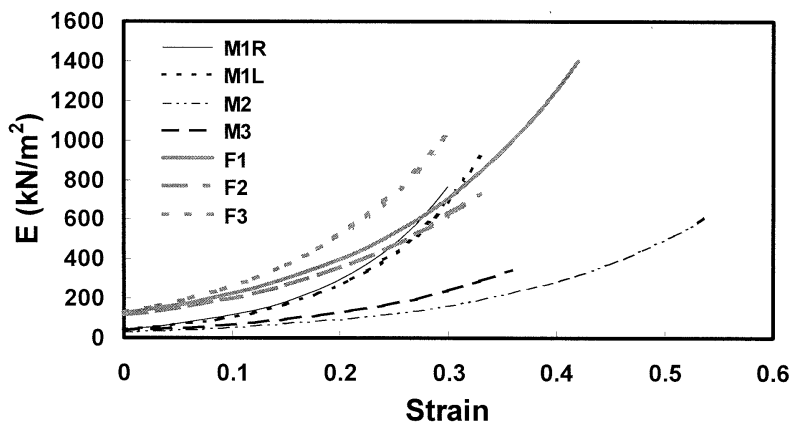

Fig. 7. Young's modulus-strain relation obtained from the differentiation of the curve in Fig. 6. regulates the vibration frequency of the VFs via the changes in length and tension of the VFs (Titze et al. 1987). There were also reports concluding that the effects of subglottic pressure on the vibration frequency of the VFs operate by the same mechanisms (Titze 1989; Hsiao et al. 1994). All the evidence indicated that the elasticity of the VFs played the major role in controlling the natural frequency of the VFs.

In the horizontal view, the vibratory movements of the VFs resembled the simple vibrating string model with fixed-ends (Titze 1994). According to eqn (1), the length of the VFs is one of the factors that affect the natural frequency of the VFs. Hollien (1960) designed a sophisticated device to measure the VF length during phonation, in which longer VF was found in higher pitch. Run and Chung (1983) used the roentgenological method to estimate the VFs length in the resting position. However, the length they measured sometimes included the arytenoid cartilage. Using clinical US, Harries et al. (1998) measured the length of VFs in boys at puberty. The above two noninvasive studies of the VF length were conducted in their resting positions without VF vibration.

In our previous study, it was verified that, for a small vibratory movement of uniform rubber string or $\mathrm{VF}$ tissue, the penetration depth of the color strip is proportional to its velocity in the direction of the US array (Shau et al. 2001). In this study, we investigated the characteristics of CDI in the fixed-ends vibrating phantom. Evidence again revealed that the vibrations of an interface between tissues with different acoustic impedances caused the color artefacts in CDI of high-frequency vibrating tissue. Along the interface, the greater the moving velocity, the deeper the color strip appeared. The length of the vibrating string could be estimated from the contour of the color image. The deviation between the experimental value and the actual length was only $3 \%$. In this study, the length of vibrating VFs was calculated by averaging the data from more than 10 frames. Thus, the error in length measurement could be minimized. From the measurements of VF length and phonation frequency, we can estimate the VFs elasticity. The mass of VF cover and its density used in our study were adopted from the previous report (Perlman and Durham 1987). To the best of our knowledge, this is the first time that both the length and the vibration frequency of the VF were measured simultaneously in human subjects under normal active phonation noninvasively and painlessly.

The relation between the tension and the length of the VFs was nonlinear, as expected in previous in vitro studies. The data were fitted very well by the exponential curves with high regression coefficients. The data taken from both VFs in the same man fell in almost the same 
curve, which supported the accuracy of the measurement.

The human VF elastic properties obtained in this study, either the tension-elongation, the stress-strain or the Young's modulus-strain relationships, were similar and compatible with the data of Perlman and Durham (1987) that were obtained in vitro. The trends of the curves and the values of the data were in about the same range. Alipour-Haghighi and Titze (1991) investigated the elastic properties of VF tissue in vitro; in the lowstrain range, the Young's moduli of the cover tissues and the curve-fitting power coefficient $(b)$ of the exponential function were $41.9 \pm 7.1 \mathrm{kPa}$ and $4.2 \pm 0.73$, respectively. Our in vivo data of men (M2 and M3) are close to those in their study.

Tran et al.(1993) and Berke and Smith (1992) reported the in vivo elasticity of human VFs cover measured under general anesthesia intraoperatively; the transverse elasticity was assessed, rather than the longitudinal. Again, the data were derived from the VFs in their resting position without active phonation. The values of longitudinal Young's moduli in the present study were 5 to 10 times higher than the transverse data obtained in the previous in vivo studies. Possible error in calculating the tension using eqn (1) may have resulted from the variation of VF mass from its nominal value. Also, the discrepancy may arise from the choice of resting VF length in the calculation of strain.

In general, we have developed a feasible method for measuring the elastic properties of human VFs in vivo under active phonation. This may provide a simple way to the understanding of phonation physiology. As stated in Baken and Orlikoff (2000), US holds a great potential for becoming a practical examination tool for assessing laryngeal functions and the stiffness inequalities between the vocal folds.

\section{SUMMARY}

Using a fixed-ends rubber vibrating string phantom, the physical interpretation of CDI artefacts on the small amplitude and high-frequency tissue vibration was studied. Because the VF vibrates as a standing sinusoidal wave in the horizontal plane, the elasticity of the VF can be estimated using the VF length and the vibrating frequency obtained at the same time during phonation. The tension and the Young's moduli of the VF measured in six adult volunteers without voice disorders varied nonlinearly with the strain. The present results agree well with the data in the literature. Because the US method is noninvasive and painless, it provides a new way for the understanding of phonation physiology and opens a possibility for the clinical evaluation of voice disorders.
Acknowledgments-This research was supported by the National Science Council, Taiwan (Grant NSC90 to 2314-B-002 to 109).

\section{REFERENCES}

Alipour-Haghighi F, Titze IR. Elastic models of vocal fold tissues. J Acoust Soc Am 1991;90:1326-1331.

Baken RJ, Orlikoff RF. Clinical measurement of speech and voice, 2nd ed. San Diego: Thomson Learning, Singular Publishing, 2000:393451.

Berke GS. Intraoperative measurement of the elastic modulus of the vocal fold. Part 1. Device development. Laryngoscope 1992;102: 760-769.

Berke GS, Smith ME. Intraoperative measurement of the elastic modulus of the vocal fold. Part 2. Preliminary results. Laryngoscope 1992;102:770-778.

Haji T, Mori K, Omori K, Isshiki N. Experimental studies on the viscoelasticity of the vocal fold. Acta Otolaryngol (Stockh) 1992a; 112:151-159.

Haji T, Mori K, Omori K, Isshiki N. Mechanical properties of the vocal fold. Stress-strain studies. Acta Otolaryngol (Stockh) 1992b;112: $559-565$.

Harries M, Hawkins S, Hacking J, Hughes I. Changes in the male voice at puberty: Vocal fold length and its relationship to the fundamental frequency of the voice. J Laryngol Otol 1998;112:451-454.

Hirano M. Morphological structure of the vocal cord as a vibrator and its variations. Folia Phoniatr 1974;26:89-94.

Hirano M. Phonosurgery. Basic and clinical investigations. Otologia (Fukuoka) 1975;21(Suppl. 1):239-262.

Hollien $\mathrm{H}$. Vocal pitch variation related to changes in vocal fold length. J Speech Hear Res 1960;3:150-156.

Hsiao TY, Solomon NP, Luschei ES, et al. Effect of subglottic pressure on fundamental frequency of the canine larynx with active muscle tensions. Ann Otol Rhinol Laryngol 1994;103:817-821.

Hsiao TY, Wang CL, Chen CN, Hsieh FJ, Shau YW. Noninvasive assessment of laryngeal phonation function using color Doppler ultrasound imaging. Ultrasound Med Biol 2001;27:1035-1040.

Kakita S, Hirano M, Ohmaru K. Physical properties of the vocal fold tissue: Measurements on excised larynges. In: Stevens K, Hirano M, ed.s. Vocal fold physiology. Tokyo: University of Tokyo Press, 1983:377-397.

Miles KA. Ultrasound demonstration of vocal cord movements. Br J Radiol 1989;62:871-872.

Min YB, Titze IR, Alipour-Haghighi F. Stress-strain response of the human vocal ligament. Ann Otol Rhinol Laryngol 1995;104:563569.

Perlman AL, Durham PL. In vitro studies of vocal fold mucosa during isometric condition. In: Baer T, Sasaki C, Harris K, ed.s. Laryngeal function in phonation and respiration. Boston, MA: College-Hill, 1987:291-303.

Perlman AL, Titze IR. Development of anin vitro technique for maesuring elastic properties of vocal fold tissue. J Speech Hear Res 1988;31:288-298.

Perlman AL, Titze IR, Cooper DS. Elasticity of canine vocal fold tissue. J Speech Hear Res 1984;27:212-219.

Run WN, Chung YS. Roentgenological measurement of physiological vocal cord length. Folia Phoniatr 1983;35:289-293.

Saito S, Fukuda H, Kitahara S, et al. Pellet tracking in the vocal fold while phonating - experimental study using canine larynges with muscle activity. In: Titze IR, Scherer RC, ed.s. Vocal fold physiology: Biomechanics, acoustics and phonatory control. Denver: Denver Center for the Performing Arts, 1983:169-182.

Shau YW, Wang CL, Hsieh FJ, Hsiao TY. Noninvasive assessment of vocal fold mucosal wave velocity using color Doppler imaging. Ultrasound Med Biol 2001;27:1451-1460.

Shau YW, Wang CL, Shieh JY, Hsu TC. Non-invasive assessment of the viscoelasticity of peripheral arteries. Ultrasound Med Biol 1999;25:1377-1388.

Tanaka S, Hirano M. Fiberscopic estimation of vocal fold stiffnessin vivo using the sucking method. Arch Otolaryngol Head Neck Surg 1990;116:721-724. 
Titze IR. The physics of small-amplitude oscillation of the vocal folds. J Acoust Soc Am 1988;83:1536-1552.

Titze IR. On the relation between subglottal pressure and fundamental frequency in phonation. J Acoust Soc Am 1989;85:901-906.

Titze IR. Principles of voice production. Englewood Cliffs: Prentice Hall, 1994:279-304.

Titze IR, Jiang J, Drucker DG. Preliminaries to the body-cover theory of pitch control. J Voice 1987;1:314-319.

Titze IR, Jiang JJ, Hsiao TY. Measurement of mucosal wave propagation and vertical phase difference in vocal fold vibration. Ann Otol Rhinol Laryngol 1993;102:58-63.
Tran QT, Berke GS, Gerratt BR, Kreiman J. Measurement of Young's modulus in thein vivo human vocal folds. Ann Otol Rhinol Laryngol 1993;102:584-591.

van den Berg J. Myoelastic-aerodynamic theory of voice production. J Speech Hear Res 1958;1:227-244.

van den Berg J, Tan TS. Results of experiments with human larynges. Pract Otorhinolaryngol 1959;21:425-450.

Yumoto E, Kadota Y. Quantitative evaluation of the effects of thyroarytenoid muscle activity upon pliability of vocal fold mucosa in anin vivo canine model. Laryngoscope 1997;107:266-272. 